\title{
Dynamic Characterization of Drosophila Antennal Olfactory Neurons Indicates Multiple Opponent Signaling Pathways in Odor Discrimination
}

\author{
Andrew S. French, Päivi H. Torkkeli, and Julia Schuckel \\ Department of Physiology and Biophysics, Dalhousie University, Halifax, Nova Scotia, Canada B3H 1X5
}

Opponent signaling refers to processes in which antagonistic signals are produced by different, but closely related, stimuli. It allows enhanced discrimination and more accurate behavioral responses. We explored opponent signaling in the Drosophila melanogaster olfactory system by measuring frequency response functions between odorant concentrations and primary olfactory neuron responses. Random fluctuations in concentration of two aliphatic and two aromatic fruit odorants were used to modulate action potentials from basiconic antennal sensilla. We separated action potentials by two-dimensional cluster analysis using amplitude and cross-correlation with a median action-potential template. Frequency response functions were fitted with either bandpass or second-order low-pass functions and then divided into two polarity groups, excitatory and inhibitory, by fitting the frequency response functions. Cluster analysis gave two, three, or four action potential clusters for each sensillum recording. Sensilla were then grouped by the patterns of response polarities of the individual neurons into four sensillum types, one with four neurons and three with two neurons. All four odorant compounds produced a mixture of excitatory, inhibitory, and null responses in different neurons. Statistical analysis of frequency response parameters for individual odorants gave only weak correlation between dynamics and some neuron types, even when comparing the dynamics of excitatory and inhibitory responses to the same odorant. However, response dynamics were significantly different between aliphatic and aromatic compounds, and between the two aliphatic compounds. Each odorant caused opposing excitatory and inhibitory signals to be sent to the antennal lobe along at least two pairs of axonal pathways.

\section{Introduction}

Understanding of the Drosophila olfactory system has advanced rapidly, including the anatomy of primary sensory sensilla, their functional roles, and molecular identification of many olfactory receptors (Stensmyr et al., 2003; Couto et al., 2005; Hallem and Carlson, 2006; Laissue and Vosshall, 2008). However, the integration of primary sensory information is not well understood (Dobritsa et al., 2003; Couto et al., 2005; Hallem and Carlson, 2006) and will probably require detailed descriptions of individual sensory neuron responses to fluctuating concentrations of odorant chemicals, including their polarities and dynamics.

Drosophila olfactory sensilla are located on the third antennal segment, morphologically divided into basiconic, trichoid, and coeloconic groups. Each sensillum contains one to four sensory neurons that send action potentials along axons into glomeruli in the antennal lobe of the brain (Couto et al., 2005; Hallem et al., 2006). Odor selectivities of individual sensory neurons are controlled, in part, by odorant receptor molecules in their distal dendrites (Couto et al., 2005; Hallem and Carlson, 2006; Hiroi et al.,

Received 0ct. 5, 2010; revised 0ct. 27, 2010; accepted Nov. 5, 2010.

This work was supported by Canadian Institutes of Health Research. We thank Shannon Meisner for excellent technical assistance.

Correspondence should be addressed to Andrew S. French, Department of Physiology and Biophysics, Dalhousie University, Halifax, Nova Scotia B3H 1X5, Canada. E-mail: andrew.french@dal.ca.

DOI:10.1523/JNEUROSCI.5243-10.2011

Copyright $\odot 2011$ the authors $\quad 0270-6474 / 11 / 310861-09 \$ 15.00 / 0$
2008). Significant attention has been focused on basiconic sensilla in the medial proximal region of the third segment (de Bruyne et al., 2001; Stensmyr et al., 2003; Hallem et al., 2004) because their sensitivity to fruit odors implies important behavioral roles and a window into central processing mechanisms.

Sensory neurons of basiconic sensilla generate background action-potential firing without olfactory stimulation (de Bruyne et al., 2001), allowing inhibitory reduction of firing in addition to the excitatory increase in firing that has been widely used to identify odorant specificity. Inhibitory responses in insect olfaction include cockroach antennal sensilla responding with opposite polarity to lemon oil (Tichy et al., 2005), Manduca sexta olfactory sensilla giving inhibitory responses to a range of odorants (Shields and Hildebrand, 2001), Drosophila basiconic and coeloconic sensilla inhibited by some food odorants and terpenes (de Bruyne et al., 2001; Yao et al., 2005), and Drosophila trichoid sensilla inhibited by fruit odorants (Hallem et al., 2004). A survey of some Drosophila olfactory receptor molecules to a wide range of odorants found $17 \%$ excitatory versus $11 \%$ inhibitory responses (Hallem and Carlson, 2006).

We showed previously that random modulation of odorant concentration, followed by measurement of input-output frequency-response functions, could determine dynamic sensitivity and response polarity of single Drosophila basiconic sensillum neurons, and that excitatory and inhibitory odorants could functionally interact at single neurons (Schuckel et al., 2009). However, that study was limited to the largest amplitude action 


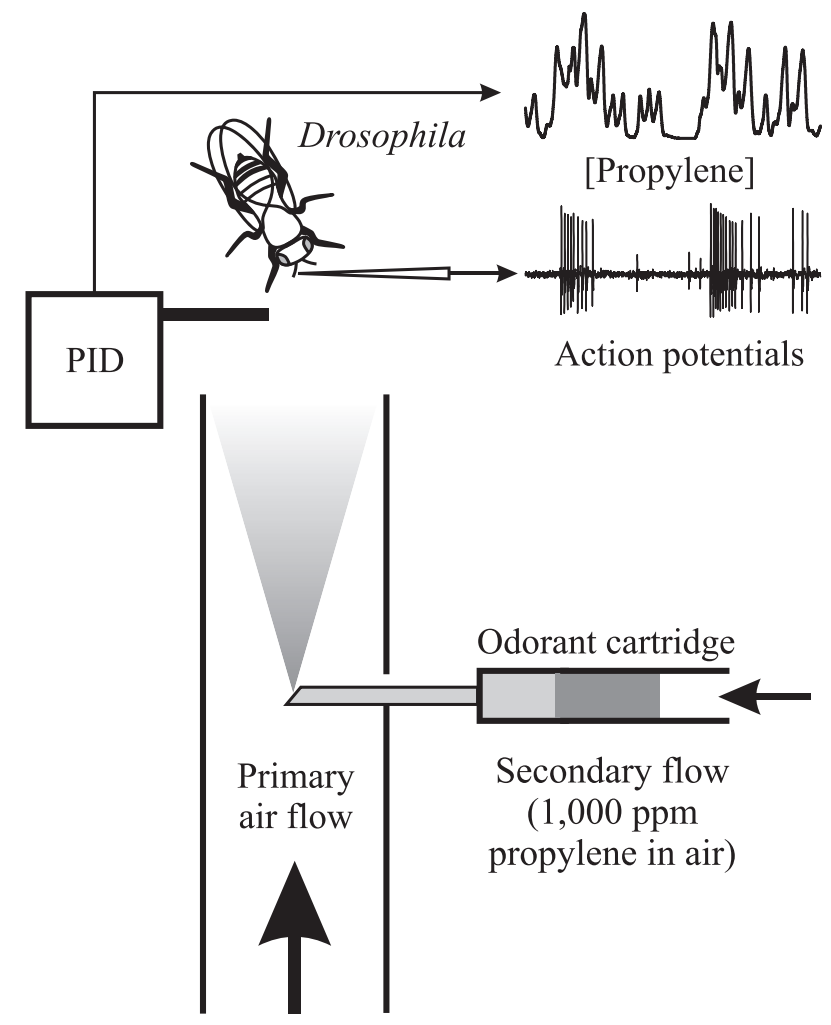

Figure 1. Experimental arrangement for random stimulation of Drosophila antennal sensilla. Primary air was driven by a fan through a 13-mm-diameter, circular flow tube made from fluorinated ethylene propylene. Secondary air containing $1000 \mathrm{ppm}$ propylene flowed over filter paper $(45 \times 15 \mathrm{~mm})$ soaked in mineral oil plus dissolved odorants. Secondary airflow was switched on and off by a two-way solenoid valve, driven by maximum-length binary sequences, and entered the primary airflow via a $16 \mathrm{~g}$ hypodermic needle. Flies were mounted in the center line of the primary airflow. Tungsten electrodes recorded action potentials from single antennal basiconic sensilla. Propylene concentration was measured by the miniature PID. Traces show parts of recordings of the binary sequence, PID signal, and action potentials during an experiment using $1 \%$ ethyl acetate.

potentials of each sensillum and made no attempt to discriminate between different sensillum groups. Here, we combined this approach with two-dimensional cluster analysis of extracellular recordings (Yang et al., 2009) to characterize dynamic responses of multiple single sensory neurons in individual basiconic sensilla. This allowed separation of sensilla into major functional groups and provided evidence of widespread excitatory versus inhibitory opponent signaling by primary olfactory neurons.

\section{Materials and Methods}

Animal preparation and electrophysiology. Flies, Drosophila melanogaster, Oregon R \#2376 (Bloomington Drosophila Stock Center) were raised and maintained in the laboratory using a standard diet (Lewis, 1960) at a temperature of $22 \pm 2^{\circ} \mathrm{C}$ under a $13 / 11 \mathrm{~h} \mathrm{light/dark}$ cycle. Flies of either sex were used within $2 \mathrm{~d}$ of hatching. Animals were held in the cut end of a $100 \mu \mathrm{l}$ plastic pipette tip. Tungsten electrodes were fabricated from 0.1-mm-diameter wire, sharpened electrolytically by passing current through the tip into concentrated potassium hydroxide solution, and pushed into the sockets of basiconic sensilla located near the proximal medial border of the posterior surface of the third antennal segment. A reference tungsten electrode was inserted into the contralateral eye. Single unit recordings were fed to a Grass P55 amplifier (Grass Technologies).

Olfactory stimulation. The stimulating system (Fig. 1) has been described previously (Schuckel and French, 2008). A fan (EVERCOOL EC4010M12CA; Cooler Guys) created the primary airflow in a $120-\mathrm{mm}-$ long, 13-mm-diameter tube made from fluorinated ethylene propylene
(Fisher Scientific). The fly was positioned within $2-3 \mathrm{~mm}$ of the exit and 2-3 mm of the tube center line. Secondary air flow came from a cylinder of compressed air containing $1000 \mathrm{ppm}$ propylene tracer gas (BOC), regulated to $20 \mathrm{kPa}$ initial pressure. It flowed through an odorant cartridge made from the shaft of a $5 \mathrm{ml}$ transfer pipette (Fisher Scientific), containing a rectangular piece of filter paper $(45 \times 15 \mathrm{~mm})$, through a two-way (open or closed) solenoid valve (Cole-Parmer 01340-02) into a $16 \mathrm{~g}$ hypodermic needle with its tip located in the center of the flow tube and $42 \mathrm{~mm}$ from the exit. The solenoid valve used PTFE materials in contact with the gas and was driven by a $24 \mathrm{~V}$ DC power supply via a photovoltaic relay to be either fully open or fully closed. The valve had a specified switching time of $5 \mathrm{~ms}$. Odorant chemicals and mineral oil were purchased from Sigma and were mixed at $20 \% \mathrm{v} / \mathrm{v}$ before final dilution to $1 \%$ or $10 \% \mathrm{v} / \mathrm{v}$. Volumes $(10 \mu \mathrm{l})$ of each mixture were loaded into separate cartridges.

Propylene concentration was measured by a miniature photoionization detector (PID, Model 200A; Aurora Scientific). The tip of the inlet probe was located directly above and within $2 \mathrm{~mm}$ of the fly antenna. The PID frequency response was $0-330 \mathrm{~Hz}$ and its concentration range was $0.05-500$ ppm propylene.

All experiments were performed at room temperature $\left(20 \pm 2{ }^{\circ} \mathrm{C}\right)$ and in a controlled humidity chamber $(<40 \%)$. The animal preparation was mounted on an air-driven, anti-vibration table. The stimulating system was mounted separately and mechanically isolated from the preparation. The stimulation system was designed to be easily disassembled and removable from the experiment without disturbing the animal preparation. The primary air tube was changed between each different odorant to avoid wall retention, and the solenoid valve was held open with fresh air flow for a period of $5 \mathrm{~min}$ between experiments. Air containing odorants was removed by a fan from the experimental area via an $80 \mathrm{~mm}$ diameter tube connected to the building exhaust system.

Experimental control and data processing. All experiments were controlled by custom-written software via a personal computer and a data acquisition board (NI6035E; National Instruments). Binary M-sequences to drive the solenoid valve were generated by the computer using a 33-bit binary shift register clocked at $10 \mathrm{~ms}$ intervals. The PID and recording electrode voltages were digitized via a 16-bit analog-to-digital converter and sampled at $0.1 \mathrm{~ms}$ intervals.

Action-potential signals were initially processed by a templatematching algorithm (Kim et al., 2004). Each $5 \mathrm{~ms}$ period in the record was used to create a variance, or energy value, and then the number of variance values plotted versus variance. The initial peak variance was assumed to be due to background noise. A computer algorithm selected the first increase in variance after the initial noise peak as the smallest action-potential variance and then detected all the nonoverlapping fluctuations in the complete record with greater variance as action potentials. The median amplitude action potential was chosen as a template for the record (Fig. 2). Cross-correlation between the template and the initial record gave a trace that could be used to select the peaks of individual action potentials by a threshold value.

Histograms of numbers of action potentials versus action potential amplitude often showed clear separation between multiple single units, but separation was significantly improved by two-dimensional (2D) cluster analysis (Yang et al., 2009). Action-potential amplitude was plotted in one dimension, and cross-correlation with the template action potential was plotted in the second dimension (Fig. 2). Separation into single unit groups was then made by operator selection of actionpotential clusters from the $2 \mathrm{D}$ plots. Single unit records were visually checked against the original recordings to verify separation.

Single unit times of occurrence were treated as Dirac delta functions of time and digitally filtered by convolution with a sinc function (French and Holden, 1971) to a bandwidth of $0-50 \mathrm{~Hz}$. The PID voltage (input) and filtered action-potential signal (output) were then resampled at 10 ms intervals. Sampled time domain data (20,000 input-output pairs) were transferred to the frequency domain using the fast Fourier transform (Cooley and Tukey, 1965) in segments of 512 sample pairs. Frequency response functions between the PID voltage and action potentials were calculated by direct spectral estimation as complex (cosine and sine) functions of frequency and plotted as Bode plots of phase and log ampli- 

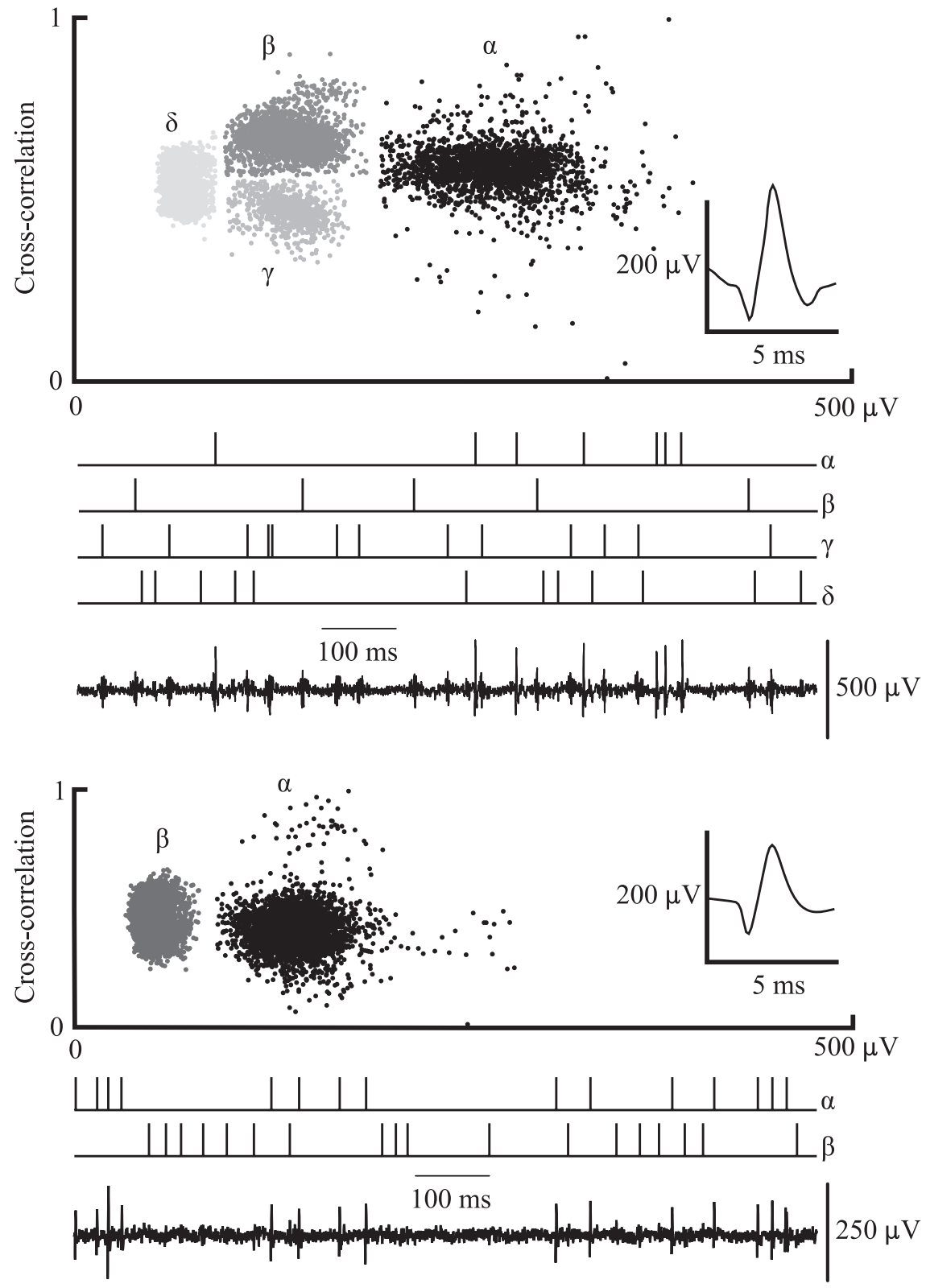

Figure 2. Separation of multiple action potentials by cluster analysis. Top, Separation of four action potentials in a sensillum; bottom, separation of two action potentials. Cluster plot abscissas are action-potential amplitude (maximum positive to maximum negative excursion) and ordinates are normalized absolute integral of cross-correlation with the median action-potential template (insets). Below each cluster plot are shown a section of the raw extracellular recording and separated action potentials.

tions. Coherence was used to estimate the information capacity, $R$, of olfactory transduction (Shannon and Weaver, 1949):

$$
R=\int \log _{2}\left[1 / 1-\gamma^{2}(\omega)\right] d \omega .
$$

Statistical analysis. Tests for significance of differences in means between distributions of fitted parameters were made using VassarStats software (http://faculty.vassar.edu/lowry/Vassar Stats.html). The Kruskal-Wallis nonparametric test was used for sets of three measurements. Tests between two variables, or between each pair when significance was detected by the Kruskal-Wallis test, were made using the MannWhitney nonparametric test. Both these nonparametric tests can be conducted directly through links at: http://faculty.vassar.edu/ lowry/ vsord.html.

\section{Results}

We used four odorants (ethyl acetate, ethyl butyrate, methyl salicylate, and phenylethyl acetate). All are naturally occurring odors that have been reported to produce both excitatory and inhibitory responses in Drosophila basiconic sensilla and odorant receptors (Hallem and Carlson, 2006; Schuckel et al., 2009). Two of the compounds are aliphatic and two are aromatic. We recorded from 30 sensilla on 28 flies ( 22 males, 6 females) to obtain a total of 223 independent single neuron recordings. No differences were detected between male and female fly responses. The four odorants were tested in random order on each preparation, but recordings were sometimes lost before we had tested all four (200 s per recording plus time to change the flow tube and odorant). Two to four different neurons were detected by cluster analysis in each sensillum.

Each frequency response function was tested for fitting by Equation 1 (bandpass) and Equation 2 (low-pass), the two parametric forms that were used previously (Schuckel et al., 2009). None of the responses had obviously different forms of frequency response function, but many could not be fitted by either equation. These had randomly scattered amplitude

tude versus log frequency (Bendat and Piersol, 1980). Frequency response functions were fitted by minimizing the coherence-weighted square error between the model and the complex data. Two model frequency response functions were used: a bandpass function:

$$
G(\omega)=\alpha j \omega \tau_{\mathrm{hi}} /\left(1-j \omega \tau_{\mathrm{hi}}\right)\left(1-j \omega \tau_{\mathrm{lo}}\right)
$$

where $G(\omega)$ is the frequency response function, $\omega$ is radial frequency, $\alpha$ is amplitude, and $\tau_{\mathrm{hi}}$ and $\tau_{\mathrm{lo}}$ are time constants; and a second-order lowpass function:

$$
G(\omega)=\alpha /\left[1+j \omega \tau \zeta-(\tau \omega / 2)^{2}\right]
$$

where $\tau$ is a time constant, $\zeta$ is a damping factor, and the other parameters are as before.

Coherence, $\gamma^{2}(\omega)$, as a function of frequency, $\omega$ (Bendat and Piersol, 1980) was calculated from the same data as the frequency response func- and phase plots, indicating no sensitivity to the odorant being applied. When fitted, frequency responses were clearly separable into excitatory or inhibitory by the polarity of the fitted amplitude, and reflected in the phase plots (Fig. 3). Positive responses had $71 \%$ bandpass and $29 \%$ low-pass dynamics. Negative responses had $16 \%$ bandpass and $84 \%$ low-pass dynamics (Table $1)$.

\section{Grouping responses by sensilla type}

To avoid premature categorization of sensilla or neurons, we used upper case Roman numerals to represent different sensilla, and lower case Greek letters to represent neurons within each sensillum in order of descending action-potential amplitude. Similar-sized action potentials were discriminated by the order of 


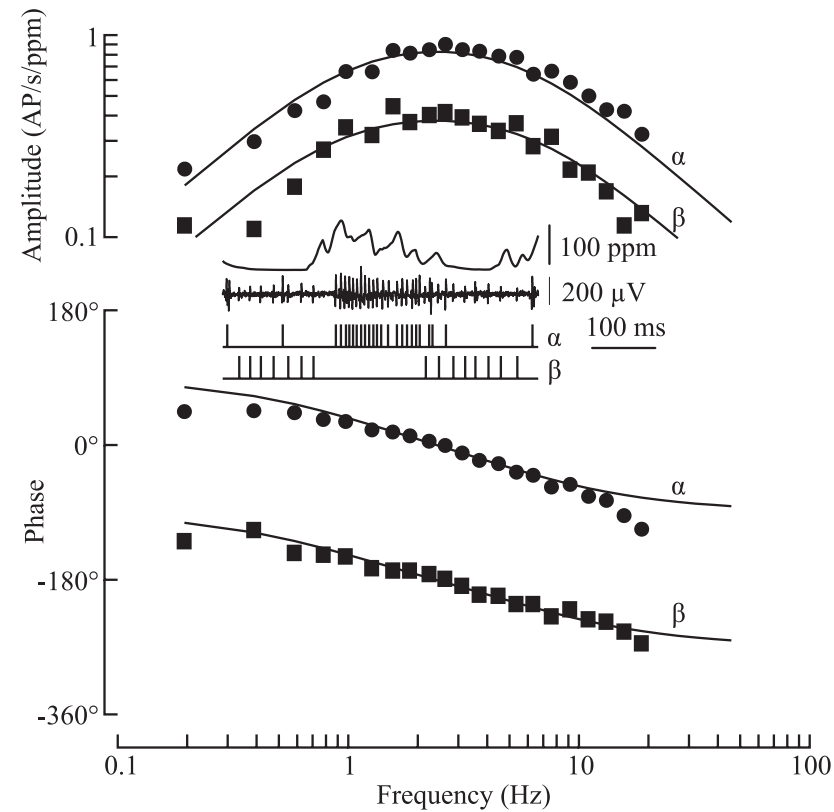

Figure 3. Frequency response functions between ethyl acetate concentration and actionpotential signals recorded from a basiconic sensillum. Propylene concentration, measured by the PID, was assumed to be linearly proportional to odorant concentration and was used as its surrogate. The total recording was of 200 s duration; the sensilla recording contained one large and one small unit, which were designated III $\alpha$ and III $\beta$, respectively. Inset, Section of the raw extracellular recording and the separated action potentials. Note the clear phase separation between the responses of the two units and the resulting separation $\sim 180^{\circ}$ between the two phase plots.

Table 1. Summary of response patterns for all neurons detected in basiconic sensilla

\begin{tabular}{|c|c|c|c|c|c|c|}
\hline \multirow[b]{2}{*}{ Odorant } & \multicolumn{2}{|c|}{ Positive (excitatory) } & \multicolumn{2}{|c|}{ Negative (inhibitory) } & \multirow[b]{2}{*}{ Unresponsive } & \multirow[b]{2}{*}{ Totals } \\
\hline & Bandpass & Low-pass & Bandpass & Low-pass & & \\
\hline Ethyl acetate & 30 & 0 & 3 & 22 & 12 & 67 \\
\hline Ethyl butyrate & 32 & 5 & 10 & 2 & 8 & 57 \\
\hline Methyl salicylate & 0 & 11 & 1 & 20 & 5 & 37 \\
\hline Phenylethyl acetate & 0 & 11 & 2 & 37 & 12 & 62 \\
\hline Totals & 62 & 27 & 16 & 81 & 37 & 223 \\
\hline
\end{tabular}

cross-correlation amplitude with the template action potential (Fig. 2). Single neuron responses were tabulated by actionpotential amplitude and response polarity to the four odorants, including unresponsive neurons which had action potentials that could not be fitted by Equation 1 or 2 (Figs. 4 and 5). Neurons that were not tested by an odorant because the recording was lost or with action potentials not detected in a recording are shown as blank entries.

Sensilla with three, and in one case four, separable neurons had similar, reliable response patterns (Fig. 4), with most responses to each odorant being the same polarity. All such sensilla were classified as type I. Three of the 86 responses did not fit this pattern. Predicted neuron responses to each odorant were made by a majority vote of the results in each column. A tie between equal numbers of responsive and unresponsive neurons in one case was decided in favor of responders.

Responses from sensilla with two detected action potentials divided clearly into three groups (types II, III, and IV) (Fig. 5). Majority voting was applied again with tie resolution as before in two cases. A summary of responses for each neuron in all four types of sensilla (Fig. 6) indicates that each of the four odorants produced both excitatory and inhibitory responses, with a major- ity being inhibitory ( 19 inhibitory vs 15 excitatory). Although the smallest action potentials in Sensillum I (Neuron I $\delta$ ) were detected in some cases, no definitive decision could be made about the sensitivity of this neuron to the four odorants from these data.

Do dynamic parameters depend on the type of sensillum? Previous studies have indicated that response polarity and dynamics are determined by the odorant receptor molecules in neurons, rather than the types of sensilla containing the neurons (Hallem et al., 2004; Hallem and Carlson, 2006). To test the hypothesis that sensillum type controls dynamics, we first looked for statistical differences between the values of fitted parameters from Equations 1 and 2 and the individual neurons, as identified by sensillum type and action-potential amplitude. We added the information capacity, $R$ (Equation 3), and the action-potential firing rate to the analysis as additional measures of olfactory transduction. Comparisons between two sets of responses were made by the Mann-Whitney test and comparisons among three sets of responses by the Kruskal-Wallis test. These nonparametric tests require a minimum of five samples in each dataset, so each of the results presented here are for datasets containing more than four measurements.

Six sets of tests for dependence of dynamic parameters on sensillum type were possible (Fig. 7). Two of the 24 tests indicated statistically significant differences between means: Neuron III $\alpha$ fired faster than $\mathrm{I} \alpha$ or II $\alpha$ in response to ethyl acetate, and its high-pass time constant, $\tau_{\text {hi }}$, was lower in response to ethyl butyrate. In each case, these differences were verified by separate Mann-Whitney tests between each pair of sensilla. No other differences between parameters in different sensilla were found.

\section{Do dynamic parameters depend on the odorants?}

To test the hypothesis that dynamics vary with odorant receptors, we combined all responses to each odorant that gave the same model fitting and tested whether dynamic parameters were equal with different odorants (Fig. 8). Parameters for bandpass excitation by ethyl acetate were significantly different from those for ethyl butyrate. However, the information capacity values, $R$, were not significantly different. We repeated this test with the data from Neuron III $\alpha$ removed, because of possible dependence on this neuron type, but the significant differences persisted.

Parameters for low-pass inhibition were available for ethyl acetate, methyl salicylate, and phenylethyl acetate. The KurskalWallis tests indicated significant differences between the dynamic parameters and the firing rates with different odorants. Follow-up Mann-Whitney tests found that dynamic parameters were different between ethyl acetate and the other two odorants, but not between methyl salicylate and phenylethyl acetate. Firing rate was lower for methyl salicylate than the other two odorants.

Low-pass excitatory responses were available for methyl salicylate and phenylethyl acetate. No significant differences were found between any of the parameters for these two odorants.

\section{Are dynamic parameters different for opposing response polarities?}

Previous work indicated that excitatory and inhibitory responses to different odorants can interact at a single neuron, which could happen at one odorant receptor or in second-messenger pathways (Schuckel et al., 2009). To examine this question further, we tested for differences between mean values of parameters for excitation and inhibition in different neurons by the same odorant. Excitatory and inhibitory responses fitted by the same equation were available for ethyl butyrate, methyl salicylate, and phenyl- 
Sensillum I

\begin{tabular}{|c|c|c|c|c|c|c|c|c|c|c|c|c|c|c|c|c|c|}
\hline & \multicolumn{3}{|c|}{ Neuron $\alpha$} & \multicolumn{3}{|c|}{ Neuron $\beta$} & \multicolumn{3}{c|}{ Neuron $\gamma$} & \multicolumn{3}{c|}{ Neuron $\delta$} \\
\hline$\#$ & EA & EB & MS & PA & EA & EB & MS & PA & EA & EB & MS & PA & EA & EB & MS & PA \\
\hline 1 & + & + & & - & $\bullet$ & + & & $\bullet$ & - & & & & & & & \\
\hline 2 & + & & & & - & & & & - & & & & & & & \\
\hline 3 & + & + & & - & $\bullet$ & $\bullet$ & & $\bullet$ & - & - & & & & & & \\
\hline 4 & + & + & & - & - & + & & - & & & & + & $\bullet$ & $\bullet$ & & \\
\hline 5 & + & + & - & - & - & + & & - & & & & + & & & & \\
\hline 6 & + & + & - & + & - & - & - & $\bullet$ & $\bullet$ & & - & $\bullet$ & & & & \\
\hline 7 & + & + & & - & - & + & & $\bullet$ & - & & & $\bullet$ & & & & \\
\hline 8 & + & + & - & - & - & + & $\bullet$ & - & & & $\bullet$ & $\bullet$ & & & & \\
\hline 9 & + & + & - & - & - & + & - & - & & + & - & - & & & & \\
\hline 10 & + & + & - & - & - & + & $\bullet$ & - & - & $\bullet$ & - & & & & & \\
\hline MV & + & + & - & - & - & + & - & - & - & $\bullet$ & - & $\bullet$ & $\bullet$ & $\bullet$ & & \\
\hline
\end{tabular}

EA - Ethyl acetate, EB - Ethyl butyrate, MS - Methyl salicylate, PA - Phenylethyl acetate, MV - Majority vote

Figure 4. Summary of fitted frequency response polarities for type I sensilla producing at least three action potentials. Sensilla were numbered $1-10$ by experimental time sequence (left column) and neurons within sensilla were labeled $\alpha-\delta$ (see Results). + , Excitatory frequency responses; - inhibitory frequency responses; $\bullet$, neurons whose frequency response functions could not be fitted. Blank entries indicate that the recording was lost or the action potential was below the noise level and not detected; + and - with no background shading indicate sensilla that did not follow the response patterns. MV indicates the majority vote for the response of the neuron to the specific odorant.

ethyl acetate (Fig. 9). The only significant difference detected was the lower firing rate seen again for neurons inhibited by methyl salicylate.

\section{Discussion}

Both inhibitory and excitatory responses have been reported in insect primary olfactory neurons (de Bruyne et al., 2001; Shields and Hildebrand, 2001; Hallem et al., 2004; Tichy et al., 2005; Yao et al., 2005) and their odorant receptors (Hallem and Carlson, 2006). The frequency response approach allowed us to detect simultaneous excitation and inhibition in multiple neurons within a sensillum during the same stimulation by fluctuating concentrations of odorants. Combining data from the four different groups of sensilla indicates that each of these four odorants causes the next stage of processing in the olfactory glomeruli to receive a different, unique combination of at least two excitatory and two inhibitory signals from these four types of sensilla alone. Extension of this model to more of the 10 different types of basiconic sensilla (Couto et al., 2005) would provide a rich source of afferent information for odor discrimination. Parallel excitatory and inhibitory, or opponent, signaling facilitates discrimination in a range of other sensory systems (Von Bekesy, 1967), such as the opponent signaling in the vertebrate visual system (Kuffler, 1953).

\section{Identification of the four sensilla types}

We recorded from the medial proximal posterior region of the antenna to observe the three large basiconic sensilla, ab1-ab3, of this region (de Bruyne et al., 2001). Of these, only ab1 contains four neurons, although a sensillum with four neurons and different odorant sensitivity has been reported in more distal regions
(Stensmyr et al., 2003). Considering the recording site and consistent three to four neurons, we assign type I to abl sensilla.

All other basiconic sensilla probably have two neurons (de Bruyne et al., 2001; Hallem et al., 2004; Couto et al., 2005), as did our types II-IV sensilla. Types ab2 and ab3 are in the same region as ab1, and the only other type that have been reported nearby are smaller ab4 sensilla. Therefore, we assign types II-IV to ab2-ab4 sensilla, but the order is complicated by the small number of odorants used here.

Functional maps of Drosophila olfactory neuron responses have been made by direct stimulation and by testing odorant receptor molecules expressed in functionally empty sensilla (de Bruyne et al., 2001; Dobritsa et al., 2003; Hallem et al., 2004; Hallem and Carlson, 2006). These data can be combined with morphological maps of odorant receptor molecules (Couto et al., 2005). However, not all receptor molecules have been tested, some originally excitatory responses are now thought to be inhibitory, and widely graded olfactory responses to single odorant puffs make it difficult to decide if some odorants are functionally detected.

Ethyl acetate excited ab2A, ab3A, and ab4A (de Bruyne et al., 2001), matching our findings. Ethyl butyrate excited ab2B (de Bruyne et al., 2001; Hallem and Carlson, 2006) matching our data for neuron II $\beta$, rather than III $\beta$ or $\operatorname{IV} \beta$, so we tentatively assign sensillum II to ab2.

The only difference between sensilla III and IV was the reliable excitation of IV $\beta$ by phenylethyl acetate compared with inhibition of III $\beta$. The only previously reported strong excitation by phenylethyl acetate was receptor OR67a in the small ab10 sensillum (Hallem and Carlson, 2006), but it is unlikely that we would have recorded multiple ab10 sensilla because they are small and were undetected in early surveys (Hallem et al., 2004). Pulsed phenylethyl acetate gave weak excitation to at least 10 different olfactory receptors (Hallem and Carlson, 2006, their supplemental material), including those found in ab2 and ab3, but not ab4, suggesting that types III and IV correspond to ab4 and ab3, respectively.

\section{Identification of neurons within sensilla}

The largest action potentials, I $\alpha$-IV $\alpha$, probably correspond to $\mathrm{ab} 1 \mathrm{~A}-\mathrm{ab} 4 \mathrm{~A}$, which assigns II $\beta-\mathrm{IV} \beta$ to ab2B-ab4B. Identification of $\mathrm{I} \beta, \mathrm{I} \gamma$, and $\mathrm{I} \delta$ is more difficult. Methyl salicylate excited ab1D (de Bruyne et al., 2001) and mildly excited OR10a, the receptor of ab1D (Hallem and Carlson, 2006, their supplemental material), whereas we only saw methyl salicylate inhibition in sensillum I. Ethyl acetate and ethyl butyrate both excited ab1A and ab1B (de Bruyne et al., 2001), whereas we found excitation of I $\alpha$ by both odorants, but inhibition of I $\beta$ by ethyl acetate (Fig. 3). Neuron I $\delta$ was only detected in two experiments and was unresponsive to all odorants. Therefore, I $\delta$ probably corresponds to ab1C, which only responds to $\mathrm{CO}_{2}$ (de Bruyne et al., 2001). I $\gamma$ would then be ab1D. Lack of functional evidence for three of the putative recep- 


\begin{tabular}{|c|c|c|c|c|c|c|c|c|c|}
\hline \multicolumn{10}{|c|}{ Sensillum II } \\
\hline$\#$ & EA & EB & MS & PA & EA & EB & MS & PA \\
\hline 11 & + & & - & - & $\bullet$ & & + & + \\
\hline 12 & + & + & $\bullet$ & - & - & + & + & $\bullet$ \\
\hline 13 & + & + & - & - & $\bullet$ & + & + & $\bullet$ \\
\hline 14 & + & $\bullet$ & - & - & - & + & + & + \\
\hline 15 & + & + & & & $\bullet$ & + & & \\
\hline 16 & + & & & - & $\bullet$ & & & + \\
\hline 17 & + & $\bullet$ & & - & - & + & & + \\
\hline 18 & + & + & & - & - & & & $\bullet$ \\
\hline 19 & + & + & - & - & - & $\bullet$ & + & $\bullet$ \\
\hline MV & + & + & - & - & - & + & + & + \\
\hline
\end{tabular}

\begin{tabular}{|c|c|c|c|c|c|c|c|c|}
\hline \multicolumn{9}{|c|}{ Sensillum III } \\
\hline & \multicolumn{4}{|c|}{ Neuron $\alpha$} & \multicolumn{4}{|c|}{ Neuron $\beta$} \\
\hline$\#$ & EA & EB & MS & PA & EA & EB & MS & PA \\
\hline 20 & + & + & - & - & - & - & + & - \\
\hline 21 & + & + & - & - & - & - & + & - \\
\hline 22 & + & + & - & - & - & - & • & • \\
\hline 23 & + & + & & - & - & - & & - \\
\hline 24 & + & + & & - & - & - & & - \\
\hline 25 & + & + & & - & $\cdot$ & - & & - \\
\hline 26 & + & - & - & - & - & $\cdot$ & + & - \\
\hline$\overline{M V}$ & + & + & - & - & - & - & + & - \\
\hline
\end{tabular}

Sensillum IV
\begin{tabular}{|c|c|c|c|c|c|c|c|c|}
\hline & \multicolumn{3}{|c|}{ Neuron $\alpha$} & \multicolumn{3}{|c|}{ Neuron $\beta$} \\
\hline$\#$ & EA & EB & MS & PA & EA & EB & MS & PA \\
\hline 27 & + & + & - & - & $\bullet$ & - & + & + \\
\hline 28 & + & + & - & - & $\bullet$ & - & + & + \\
\hline 29 & + & + & - & - & - & - & + & + \\
\hline 30 & + & + & & - & - & - & & + \\
\hline MV & + & + & - & - & - & - & + & + \\
\hline
\end{tabular}

EA - Ethyl acetate, EB - Ethyl butyrate, MS - Methyl salicylate, PA - Phenylethyl acetate, MV - Majority vote

Figure 5. Summary of fitted frequency response polarities for types II-IV sensilla producing two action potentials. Labeling is the same as Figure 4 . Sensilla were separated into the three different types by their patterns of response polarities so that no column contained both excitatory and inhibitory responses.

tors in ab1 (OR92a, OR42b, and GR21a) prevents more definite assignments.

\section{Olfactory response dynamics}

One aim of our work is to discover which stage or stages of olfactory transduction control the dynamics of odorant responses. Our data support the idea that receptor dynamics are tightly linked to odorant receptor molecules. The bandpass and lowpass functions that we used to fit the data (Eqs. 1, 2) were not intended to represent real physical processes. They merely provide a mechanism for mapping a large number of measurements (cosine and sine amplitudes vs frequency) by a small number of parameters (time constants and damping factors) that can efficiently represent the dynamic behavior. Of course, it is possible to speculate about physical processes that might underlie these frequency response functions, such as high-frequency time con- stants being related to binding of odorants to receptor molecules and/or odorant binding proteins.

Measurements of responses from six different neurons with four different odorants gave only weak statistical evidence for any relationship between response dynamics and sensilla (Fig. 7), but there was strong evidence that responses to ethyl acetate and ethyl butyrate had different dynamics from each other and from the two aromatic compounds (Fig. 8). These findings support previous suggestions that response dynamics are linked to odorant receptors, rather than sensilla or individual neurons (Hallem and Carlson, 2006). This linkage could reflect properties of the odorant receptors themselves, but could also reflect the behavior of associated processes, including odorant binding proteins and second-messenger pathways.

The finding that response dynamics did not depend on polarity (Fig. 9) is particularly interesting because there are many different olfactory receptors in different neurons, and possibly different intracellular signaling pathways that follow receptor activation, suggesting that some process at, or very close to, the receptor molecule controls dynamics. There are also significant implications for models of olfactory receptors as directly activated cation channels (Sato et al., 2008), because any realistic transduction model must account for both excitatory and inhibitory responses, which have now been found at both the receptor and neuron levels.

\section{Information capacity and signal-to-noise levels}

The information capacity parameter, $R$, did not depend on sensillum, neuron, odorant, or response dynamics. This parameter estimates the amount of information that could be carried by the neuron and is independent of signal amplitude. We derived $R$ from coherence, which depends on signal-to-noise ratio and/or nonlinearity. The constant value of $R$ within each group of experiments probably means that the sensory neurons all have similar general structure and function, with approximately the same noise level, and similar nonlinear contributions from stages such as action-potential encoding. The range of information capacity values ( $\sim 3-15 \mathrm{Bits} / \mathrm{s})$ is lower than the value of $\sim 200 \mathrm{Bits} / \mathrm{s}$ typically observed in spiking mechanoreceptors (Juusola and French, 1997), but not unreasonable, given the smaller available bandwidth, less controlled input signal, and more demanding recording conditions.

\section{Random versus pulse stimulation}

Functional characterization of insect olfactory neurons has most commonly used pulsed odorant concentration, combined with action potential counting. In the frequency domain this corresponds to a brief stimulus with very high frequencies at the leading edge of the pulse, followed by a longer duration with constant or zero frequency stimulation. Although useful dynamic information has been obtained from pulsed odorant application, the relatively brief duration of the experiment, combined with strong nonlinearities, such as threshold and saturation, is not optimal for characterizing dynamic responses. For example, dependence of dynamics on stimulus amplitude (Hallem and Carlson, 2006) is nonlinear behavior that may not be important with more natural stimuli.

In contrast, random stimulation provides a wide frequency range over an arbitrarily long duration, and is arguably closer to the natural situation of a fly encountering fluctuating concentrations of odorants as it navigates through a turbulent atmosphere containing multiple odorant sources. Random stimulation also 

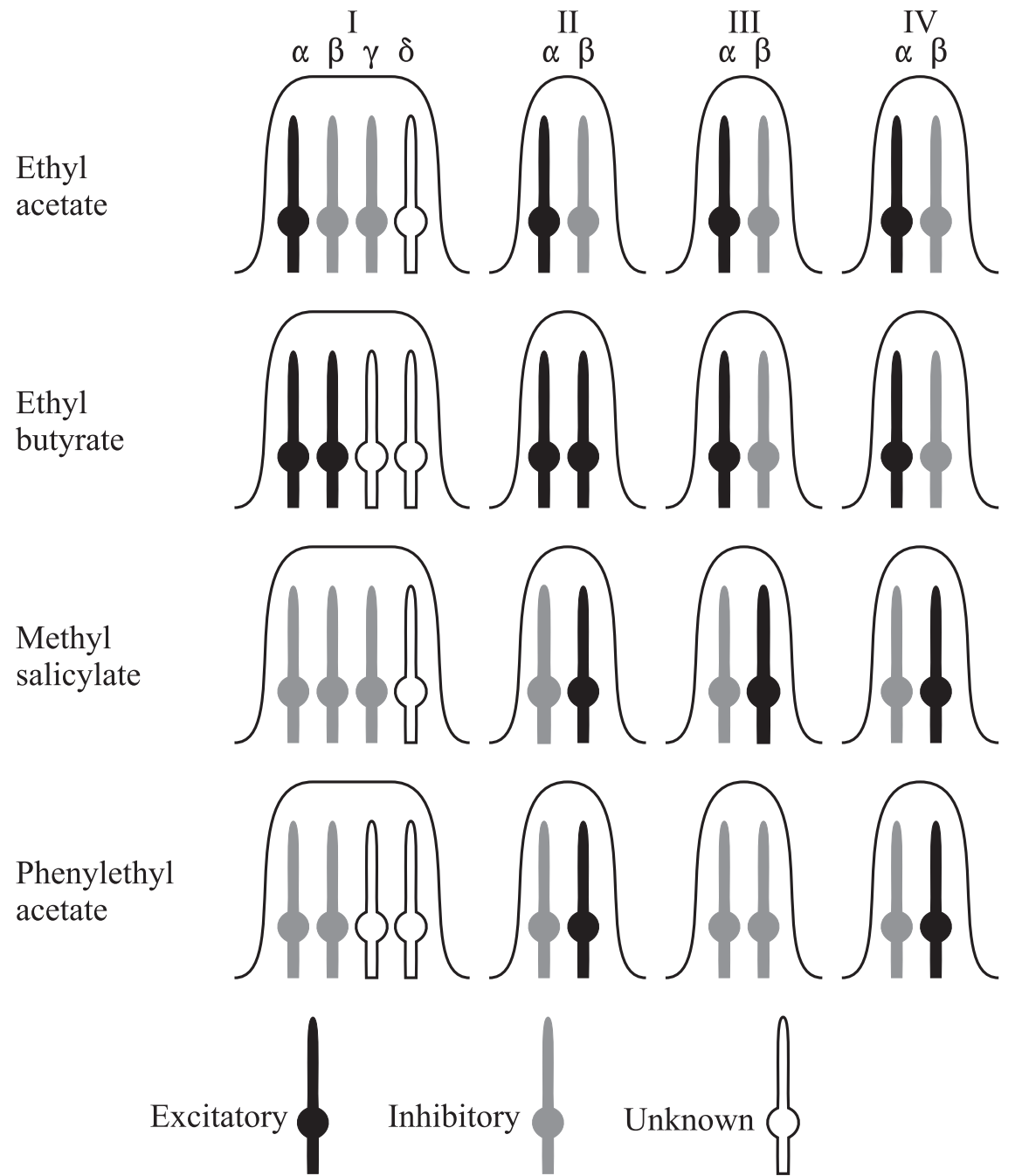

Figure 6. Summary of frequency response polarities of the different neurons within each of the four types of sensilla to each of the four odorants tested. Excitatory and inhibitory responses are indicated by shading. Unshaded neurons had no detected responses to the individual odorants. Note that each odorant would cause a different pattern of excitatory and inhibitory responses to be sent to the olfactory glomeruli.

has a linearizing effect (Spekreijse and Oosting, 1970) because a large amplitude component at one frequency may keep the neuron firing and allow the rate of firing to be modulated by a smaller component that would otherwise be too weak to cross the threshold. This has led to the concept of increased sensory information transmission by stochastic resonance in sensory encoding (Douglass et al., 1993).

\section{Olfactory coding}

Mechanisms of odor discrimination probably involve some form of template matching, in which patterns of primary olfactory neuron activity produce specific responses in neural networks with corresponding interconnections. General models of this type have recently been described for insect pheromone and food odor discrimination (Martin and Hildebrand, 2010). Such models usually include local inhibitory interneurons within and between olfactory glomeruli. However, the prevalence of mixed excitatory and inhibitory responses to different odorants in olfactory neurons is becoming clear and suggests more direct mechanisms of discrimination by selecting patterns of excitatory and inhibitory inputs from primary sensory neurons.
To illustrate this approach, we can consider a network receiving inputs from all of the 10 known basiconic sensilla in Drosophila, i.e., 22 neurons. If most of the primary neurons were either excited or inhibited in different combinations by odors, and the network reached decisions based on these purely binary inputs, the possible number of combinations would approach $2^{20}$, or more than a million. Including relative response amplitude information and/or response dynamics would enormously increase discriminative power.

\section{References}

Bendat JS, Piersol AG (1980) Engineering applications of correlation and spectral analysis. New York: Wiley.

Cooley JW, Tukey JW (1965) An algorithm for the machine calculation of complex Fourier series. Math Comput 19:297-301.

Couto A, Alenius M, Dickson BJ (2005) Molecular, anatomical, and functional organization of the Drosophila olfactory system. Curr Biol 15:1535-1547.

de Bruyne M, Foster K, Carlson JR (2001) Odor coding in the Drosophila antenna. Neuron 30:537-552.

Dobritsa AA, van der Goes van Naters W, Warr CG, Steinbrecht RA, Carlson JR (2003) Integrating the molecular and cellular basis of odor coding in the Drosophila antenna. Neuron 37:827-841.

Douglass JK, Wilkens L, Pantazelou E, Moss F (1993) Noise enhancement of information transfer in crayfish mechanoreceptors by stochastic resonance. Nature 365:337-340.

French AS, Holden AV (1971) Alias-free sampling of neuronal spike trains. Kybernetik 8:165-171.

Hallem EA, Carlson JR (2006) Coding of odors by a receptor repertoire. Cell 125:143-160.

Hallem EA, Ho MG, Carlson JR (2004) The molecular basis of odor coding in the Drosophila antenna. Cell 117:965-979.

Hallem EA, Dahanukar A, Carlson JR (2006) Insect odor and taste receptors. Annu Rev Entomol 51:113-135.

Hiroi M, Tanimura T, Marion-Poll F (2008) Hedonic taste in Drosophila revealed by olfactory receptors expressed in taste neurons. PLoS One 3:e2610.

Juusola M, French AS (1997) The efficiency of sensory information coding by mechanoreceptor neurons. Neuron 18:959-968.

Kim S, McNames J, Burchiel K (2004) Action potential detection with automatic template matching. Conf Proc IEEE Eng Med Biol Soc 1:41-44.

Kuffler SW (1953) Discharge patterns and functional organization of mammalian retina. J Neurophysiol 16:37-68.

Laissue PP, Vosshall LB (2008) The olfactory sensory map in Drosophila. Adv Exp Med Biol 628:102-114.

Lewis EB (1960) A new standard food medium. Drosophilia Inform Serv 34:117-118.

Martin JP, Hildebrand JG (2010) Innate recognition of pheromone and food odors in moths: a common mechanism in the antennal lobe? Front Behav Neurosci 4:159.

Sato K, Pellegrino M, Nakagawa T, Nakagawa T, Vosshall LB, Touhara K (2008) Insect olfactory receptors are heteromeric ligand-gated ion channels. Nature 452:1002-1006.

Schuckel J, French AS (2008) A digital sequence method of dynamic olfactory characterization. J Neurosci Methods 171:98-103.

Schuckel J, Torkkeli PH, French AS (2009) Two interacting olfactory trans- 

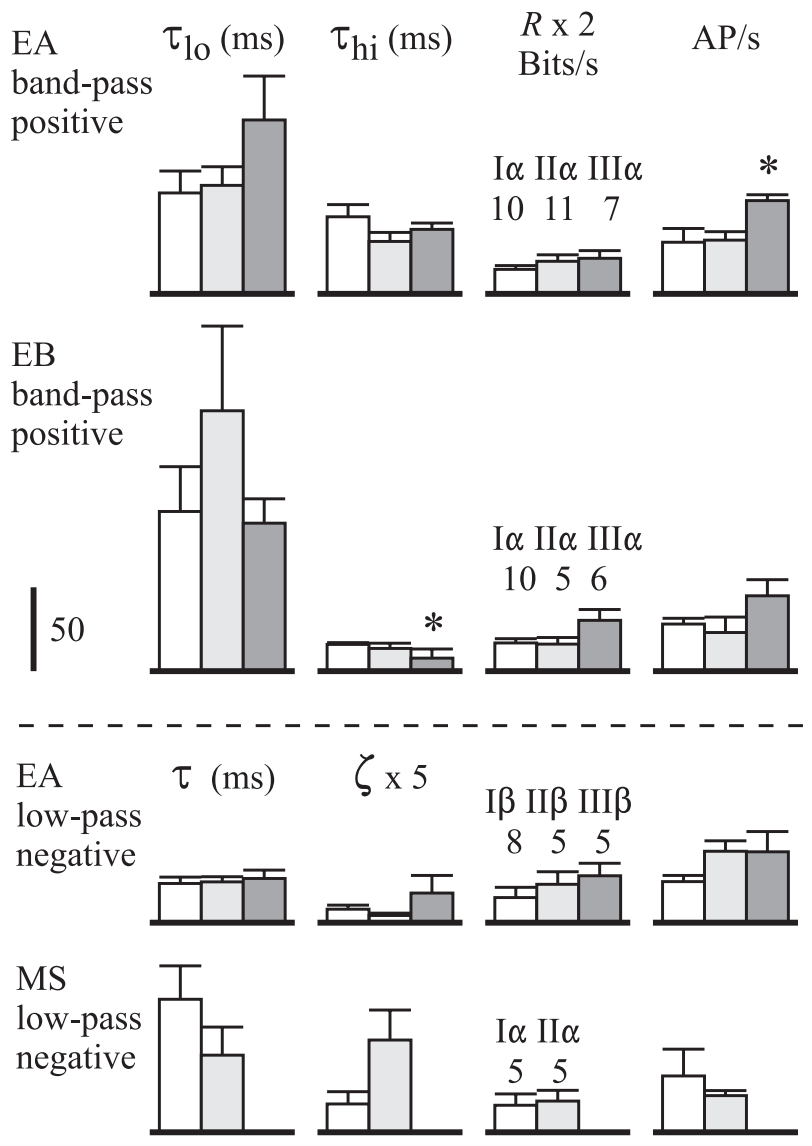

PA

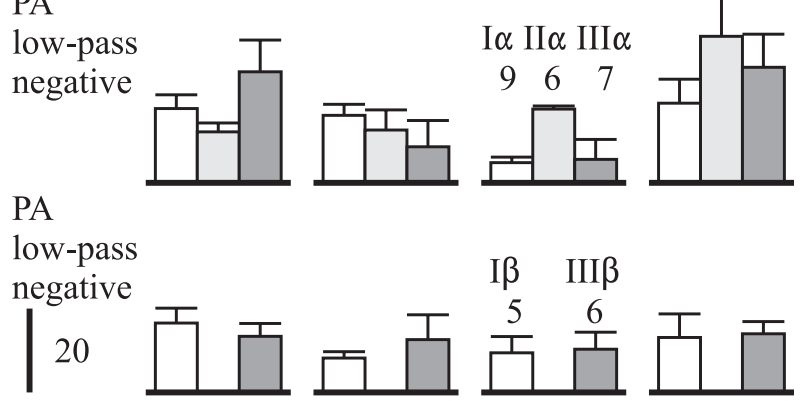

Figure 7. Comparison of dynamic parameters from neurons in different sensilla to the same odorant. Bars show means and SEs for fitted parameters of Equations 1 and 2. Each horizontal row shows data from the same two to three sensilla, identified at the information capacity $(R)$ values, together with the numbers of measurements. Top, bandpass data; bottom, low-pass data. EA, Ethyl acetate; EB, ethyl butyrate; MS, methyl salicylate; PA, phenylethyl acetate. Asterisks indicate the only two significant differences found in the 24 sets: slower action potential firing to ethyl acetate by Neuron III $\alpha$ and lower high-frequency time constant to ethyl butyrate by Neuron III $\alpha$. ${ }^{*} p \leq 0.05$.

duction mechanisms have linked polarities and dynamics in Drosophila melanogaster antennal basiconic sensilla beurons. J Neurophysiol 102:214-223.

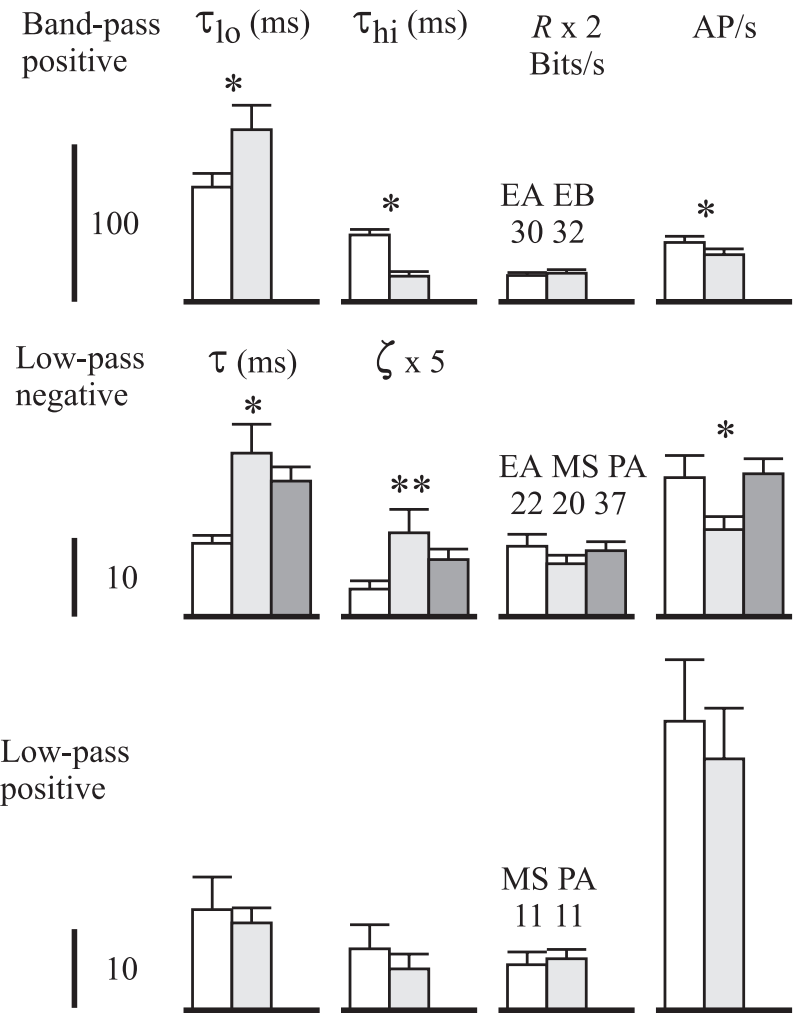

Figure 8. Statistical tests for differences between dynamic parameters from neurons stimulated by different odorants. Each row compares two to three odorants that produced the same form of response in sufficient numbers of recordings to perform tests. Labeling as for Figure 7, but with odorants and numbers of measurements identified above the information capacity data. Dynamic parameters and action potential firing rates were all significantly different between ethyl acetate and ethyl butyrate, and between ethyl acetate and either methyl salicylate or phenylethyl acetate, but not between methyl salicylate and phenylethyl acetate. EA, Ethyl acetate; $E B$, ethyl butyrate; $M S$, methyl salicylate; $P A$, phenylethyl acetate. ${ }^{*} p \leq 0.05,{ }^{*} p \leq$ 0.01

Shannon CE, Weaver W (1949) The mathematical theory of communication. Urbana, Illinois: University of Illinois.

Shields VD, Hildebrand JG (2001) Recent advances in insect olfaction, specifically regarding the morphology and sensory physiology of antennal sensilla of the female sphinx moth Manduca sexta. Microsc Res Tech 55:307-329.

Spekreijse H, Oosting H (1970) Linearizing: a method for analysing and synthesizing nonlinear systems. Kybernetik 7:22-31.

Stensmyr MC, Giordano E, Balloi A, Angioy AM, Hansson BS (2003) Novel natural ligands for Drosophila olfactory receptor neurones. J Exp Biol 206:715-724.

Tichy H, Hinterwirth A, Gingl E (2005) Olfactory receptors on the cockroach antenna signal odour ON and odour OFF by excitation. Eur J Neurosci 22:3147-3160.

Von Bekesy G (1967) Sensory inhibition. Princeton, New Jersey: Princeton UP.

Yang Z, Zhao Q, Liu W (2009) Improving spike separation using waveform derivatives. J Neural Eng 6:046006.

Yao CA, Ignell R, Carlson JR (2005) Chemosensory coding by neurons in the coeloconic sensilla of the Drosophila antenna. J Neurosci 25:8359_ 8367. 
EB $\quad \tau_{\text {lo }}(\mathrm{ms}) \quad \tau_{\mathrm{hi}}(\mathrm{ms}) \quad R \times 2 \quad \mathrm{AP} / \mathrm{s}$

band-pass

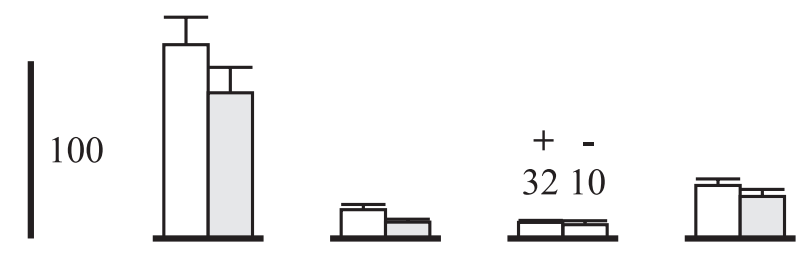

MS $\tau(\mathrm{ms}) \quad \zeta \times 5$

low-pass
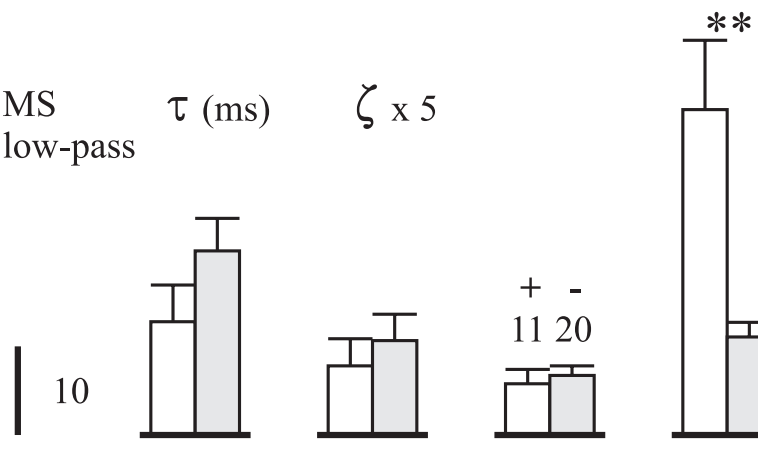

PA

low-pass
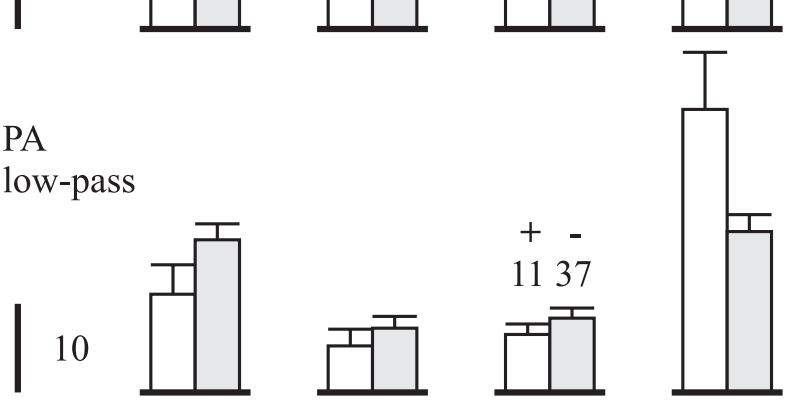

Figure 9. Statistical tests for differences between dynamic parameters from neurons giving excitatory responses and other neurons giving inhibitory responses to the same odorant. Each row compares excitatory $(+)$ and inhibitory $(-)$ responses to the same odorant, identified with the numbers of measurements above the information capacity data. Other labeling as for Figure 7. EB, Ethyl butyrate; $M S$, methyl salicylate; $\mathrm{PA}$, phenylethyl acetate. The only significant difference was in the firing rate data for methyl salicylate. ${ }^{* *} p \leq 0.01$. 